\title{
A randomized observational analysis examining the correlation between patients' food sensitivities, micronutrient deficiencies, oxidative stress response and immune redox status
}

\author{
Irena Steele, Daniel Allright, Roger Deutsch
}

Research and development Department, Cell Science Systems, 852 S Military Trail, Deerfield Beach, FL 33442, USA

Corresponding Author: Irena Steele, PhD. Head of the Research and development Department, Cell Science Systems, 852 S Military Trail, Deerfield Beach, FL 33442, USA

Submission Date: February $5^{\text {th }}, 2020$; Acceptance Date: March 26 ${ }^{\text {th }}$, 2020; Publication Date: March $30^{\text {th }}, 2020$

Citation: Steele I., Allright D., Roger Deutsch R. A randomized observational analysis examining the correlation between patients' food sensitivities, micronutrient deficiencies, oxidative stress response and immune redox status. Functional Foods in Health and Disease. 2020; 10(3): 127138. DOI: https:/doi.org/10.31989/ffhd.v10i3.695

\section{ABSTRACT}

Background: Malnutrition due to insufficient intake of micronutrients, or due to impaired delivery of micronutrients to patients' cells, is suppressing immune functions that are fundamental to host protection. Concurrently, an excessive triggering of patients' immune reactions as the result of adverse responses to certain food antigens, can also lead to various chronic health conditions.

Objective: To examine nutritional and immunological status in patients' groups varying in age, dietary regimens and gastrointestinal condition; and explore a possible correlation between an impaired patients' immune status and micronutrient deficiencies, food sensitivity and oxidative stress responses.

Methods: This is a population-based study consisting of a American residents, age 13 and older, who completed the investigator's provided questionnaires with application of cell-based individualized functional assays. Data for this paper were collected from 845 individuals between May and September 2019, as part of CSS CNA beta study. Micronutrient deficiencies, immune Redox status, antioxidative responses and food sensitivity profiles were assessed for each patient participating in this study.

Results: The group of patients with low Redox status demonstrated significantly higher percent of immune reactivity (17\%) to food antigens as compared to $15 \%$ reactivity detected in the groups with the average and strong Redox response. An average number of identified micronutrient 
deficiencies, as well as beneficial anti-oxidative protective compounds, was also significantly higher in the group with the weak immune function as compared to other two groups.

Conclusion: This study suggests that high food sensitivity is associated with a higher nutrient deficiency, a stronger oxidative stress response and a lower immune redox status.

\section{BACKGROUND}

Micronutrient deficiencies are recognized as a global public health issue; and poor nutritional status compromises immune function thus predisposing to many health issues. The immune system acts to protect the host from infectious agents that exist in the environment (bacteria, viruses, fungi, parasites) and from other harmful insults such as removal of toxins and cellular debris. It relies on two functional branches: the innate and the acquired; both involving a diversity of blood-borne factors (complement, antibodies, and cytokines) and cells (macrophages, polymorphonuclear and mononuclear cells; and, lymphocytes). The adequate functioning of this defensive system is critically impacted by intracellular nutritional status, and its interaction with the host' cells. Lacking adequate nutrition, the immune system is clearly deprived of the components needed to generate an effective immune response. Various micronutrients are essential for immune competence, particularly vitamins A, C, D, E, B2, B6, and B12, folic acid, iron, selenium, and zinc [1], [2], [3], [4]. For example, vitamin A deficiency impairs mucosal barriers and diminishes the function of neutrophils, macrophages, and NK cells [5]. Antioxidant nutrients play a pivotal role in maintaining the antioxidant/oxidant balance in immune cells, in protecting them from oxidative stress and preserving their adequate function, such as protection from DNA breaks, oxidative lesions, or both [6], [7]. Oxidative stress is worsened in infection if micronutrients are deficient. Leukocyte functions are strongly influenced by the antioxidant/oxidant balance, because immune cells produce reactive oxygen species (ROS) to support their functions [8]. Thus, adequate amounts of neutralizing antioxidants are required to prevent damage to the immune cells themselves. Investigations in humans confirm a lower capacity of lymphocytes deficient in micronutrients/ antioxidants to proliferate in response to mitogen activation.

Immune function may be improved by restoring deficient micronutrients to recommended levels, thereby increasing resistance to infection and supporting faster recovery when infected. Therefore, the ability to identify the key antioxidants that appears to be deficient in patients' cells, offers a valuable tool to strengthen patients' immune system and prevent/ improve many health conditions [9]. Nutrient repletion via supplements and/or food sources can restore immune function and resistance to infection. However, excessive amounts of some nutrients also impair immune function [10] and in certain cases potential harmful effects of excessive supplementation have been observed in some clinical trials [11]. Thus, Ferrante et al., reported that dietary intake of long-chain n-3 polyunsaturated fatty acids was associated with selective depression of circulating natural killer cells of the patients [12].

Additional complexity in assessing individual nutritional requirements and patients' immune responses is compounded by the individual reactions of immune system to food antigens. The initiation of adverse immune responses to food may appear from the development of a 'new' reaction to a food antigen and/or a loss of previously acquired tolerance. This ability of the immune system to discriminate between harmful and 'safe' substances is the result of complex interactions between the cells which sample the gut contents and 'show' it to the immune system (antigen 
presenting cells) and the immune cells. The signals which the immune cell receives during the encounter with the antigen presenting cell will dictate whether the immune cell will be activated or remain quiescent. The loss of this state of tolerance results in an adverse immune response to a food antigen. Therefore, the proper and timely identification of these reactive foods is critical to maintenance of homeostasis and prevention of pathological inflammatory responses.

All these data suggest that nutritional supplementation should be tailored to each patient, which is possible by using a functional assessment of the patients' cells. For at least the last three decades cellular assays have been used as a sensitive functional approach in the assessment of a patient's nutritional status. In the present study we used the cellular functional response assay (Cellular Nutrition Assay) developed by Cell Science Systems, Corp. (Deerfield Beach, Florida) to evaluate a possible correlation between nutritional deficiencies, food sensitivity profiles and their immune status in groups that vary in age, life style, dietary habits, supplementation and gastrointestinal symptoms.

\section{METHODS}

\section{Tested Groups}

This study was designed as a randomized observational analysis including a large number (845) of consecutive patients that volunteered to donate venous blood and complete a questionnaire on lifestyle, dietary habits, and self-reported health conditions. Informed consent for anonymous reporting of outcomes was obtained from each subject. Study subjects were self-selected in that they requested, through their health care provider, to have their blood drawn for identification of food and chemical sensitivities through Alcat testing. Approximately $15 \mathrm{cc}$ of venous blood was taken, in addition to that required for the already requested lab test, and the investigators selfdetermined that this condition posed no additional risk to patients. The study population was represented by different ages (18- 86 years old), genders, medical and ethnic backgrounds. Subjects were asked to complete questionnaires regarding their biological and medical background, lifestyle, and diet. Subjects use of anti-inflammatory medications were discontinued, if possible, for a minimum of 72 hours prior to blood draw. Finally, all subjects were divided into the groups of interest. The subjects' personal data were anonymized and secured, in accordance with HIPAA regulations, until the lab data findings were complete. Each patient received their respective lab test results.

\section{Redox Assay for Immune Function Status Assessment}

The redox status of patients was measured using the Cell Science Systems (CSS) Redox assay. Redox is a measurement of overall antioxidant function of patients' peripheral immune cells. Briefly, the patients' leucocytes suspended in the minimal media, containing patients' autologous serum, are challenged with increasing concentrations of a free radical generating system $\left(\mathrm{H}_{2} \mathrm{O}_{2}\right)$. The cells' ability to resist oxidative damage, as determined by lymphocyte count, was assessed and gradated by CSS Redox software.

\section{Micronutrient Assay for Micronutrients Deficiencies Assessment}

All 845 subjects were tested for micronutrient deficiencies (MN) using the CSS Micronutrient Deficiency assay (Cellular Nutritional Assay - CNA). Briefly, patients' serum and a mixture of lymphocytes were isolated from the whole blood of patients. Patients' cells were resuspended in 
autologous serum and cultured in the presence of different micronutrients, while stimulated by mitogen. The lymphocytes' growth rate, without the addition of a micronutrient, is defined as the patient's baseline. Results were expressed as the stimulation index (SI) which is the percentage of stimulation in response to mitogen, with non-stimulated values being $100 \%$. An increase in lymphocyte count following the addition of a specific micronutrient is regarded as indicating a functional deficiency of the specific micronutrient. The number of micronutrient deficiencies for each patient was recorded. An average number of deficiencies per group was calculated.

\section{Antioxidant Assay for Antioxidant Deficiencies Assessment}

CSS cellular antioxidant test (AO) was used to identify patient-specific antioxidants that may be particularly protective in resisting oxidative stress and restoring antioxidant function. Similar to the MN testing, patients' serum and a mixture of lymphocyteswere isolated from the whole blood of patients. Patients' cells resuspended in autologous serum were grown in the presence of different antioxidants, while exposed to $\mathrm{H}_{2} \mathrm{O}_{2}$ at the concentration that was determined to create oxidative stress to the patients' lymphocytes in vitro. The lymphocytes' growth rate without additional antioxidants, is identified as the patient's baseline. Results are expressed as the

stimulation index, which is the percentage of stimulation of cell proliferation in the presence of single antioxidant, compared with the proliferation rate of cells without addition of antioxidants being $100 \%$. The number of antioxidant deficiencies (AOD) for each patient was recorded. An average number of deficiencies per each group was calculated.

\section{Alcat Assay for Food Sensitivities Assessment}

Food sensitivity profiles of all 845 subjects were assessed by Alcat 3D CSS assay. Briefly, the Alcat Test is a leukocyte activation test that measures cellular immune response, such as changes in cell size and cell number following the exposure to individual food substances in vitro. Cellular changes in leukocytes were detected by impedance technology (ROBOCat II) and analyzed by the Alcat software. Total number of reactions was expressed as percent of reactivity. Average percent of reactivity per group was calculated and compared between Redox groups.

\section{Statistical Analysis}

Repeated-measures analysis of variance (ANOVA) tested for within- and between-group differences in MND, AOD and Alcat \% of Reactivity for groups that vary in age, diet, smoking and alcohol consumption habits, and GI status. Statistical differences were determined by t-test or by one-way analysis of variance (ANOVA), using GraphPad Prism V.8.2.1, 2019. A p value < 0.05 was considered to indicate a significant difference between values, while a $\mathrm{p}$ value $<0.10$ was regarded as marginal difference.

\section{RESULTS}

Evaluation of Alcat Reactivity, Micronutrient Deficiency and Antioxidant Deficiency in Subjects with Various Redox Status (RS).

First, using CSS Redox Assay we evaluated level of RS in each subject. Of the 845 tested subjects, 101 demonstrated a weak RS, 189 fell into a strong RS group, while 555 reported an average RS 
response. These groups were selected for the further assessments, such as Alcat 3D food sensitivity testing, CNA Micronutrients Deficiency assay (MND) and CNA Antioxidant

\section{Protection assay (AOP).}

Alcat Reactivity results were expressed as an average percent of reactivity (AvgAR). AvgAR was significantly increased in the weak redox group comparative to the other two groups: $\mathrm{F}(2,842)=$ 6.342, $\mathrm{p}=0,002$ (Fig 1, A).

Moreover, average numbers of micronutrient and antioxidant deficiencies were significantly higher in the weak redox group comparative to the average and strong redox groups with $\mathrm{F}(2,859)$ $=4.720, \mathrm{p}=0.0091$ and $\mathrm{F}(2,859)=4.640, \mathrm{p}=0.0099$ respectively (Fig 1, B and C).
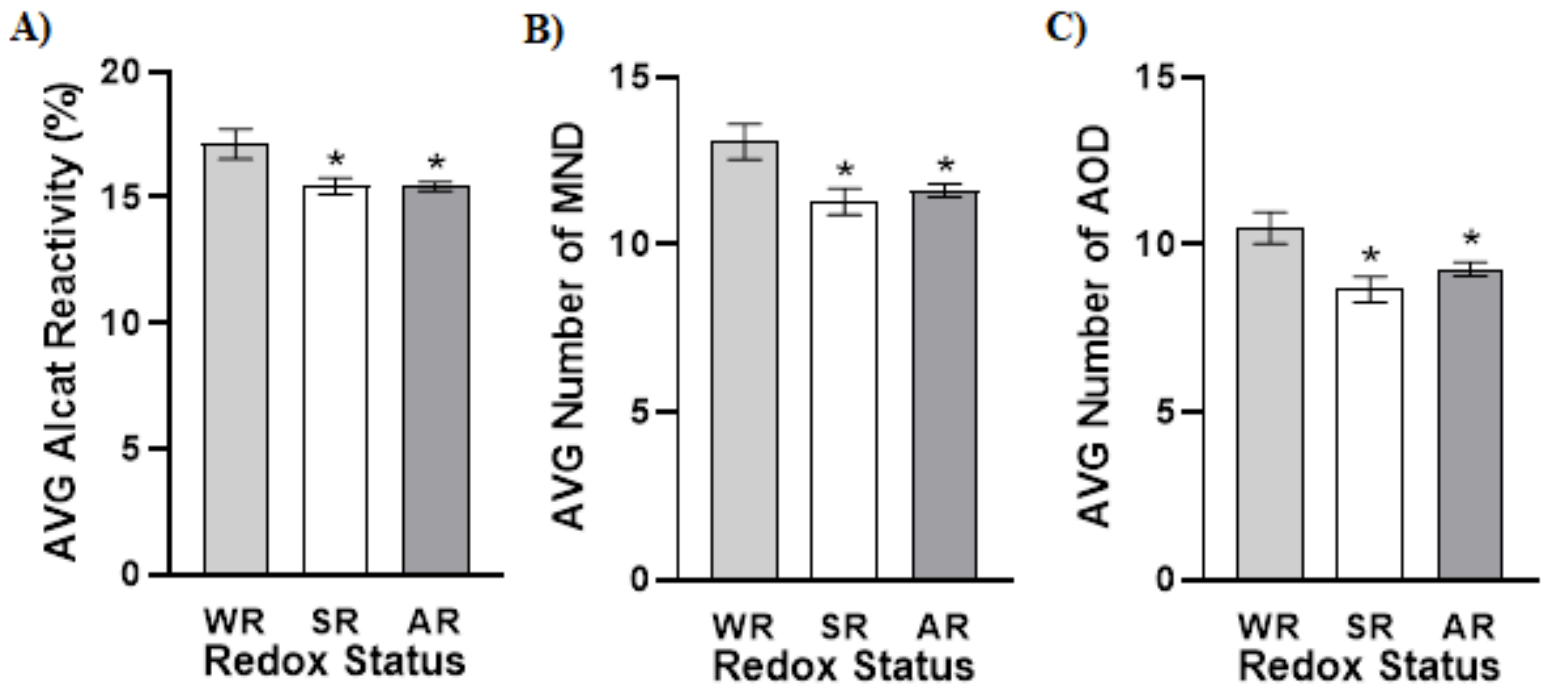

Figure 1: Participants with a weaker redox status demonstrated overall higher Alcat reactivity (A), MND (B), and AOD (C) across the study population $(n=845)$. Data shown are participant's blood samples subdivided into weak reactions (WR; $n=101$ ), Strong reactions $(S R ; n=189$ ), and Average reactions ( $A R ; n=555)$, based on the assessment of their redox assay. A one-way ANOVA indicated that the average percent of reactivity was significantly increased $(\mathrm{p}=0.002)$, post hoc analysis with Turkey's test indicated the WR group was significantly different from both the AR and SR groups $(\mathrm{p}=0.001$ and 0.007 , respectively). Data are shown as the group average \pm SEM; asterisks indicate significance when compared against the other groups.

\section{Effect of alcohol on MND, AOD, redox status and food reactivity responses}

There was a larger percentage of strong redox responses (20\%) in the group with low consumption of alcohol as compared to a high consumption alcohol group (12\%), while high consumption alcohol group had a 7\% higher average number of weak redox responses, as compared to the low consumption alcohol group. According to the chi-square test, high alcohol consumption had marginal negative effect on immune redox function of the tested subjects $(p=0.07)$ (Fig 2, A). An average percent of reactivity towards food antigens was significantly higher in the LAC group than in the HAC group ( $\mathrm{p}=0.045$ ) (Fig. 2, B). There was no significant difference in the number of micronutrient and antioxidant deficiencies between low and high alcohol consumption groups as assessed by the one-way ANOVA analyses. 
A)

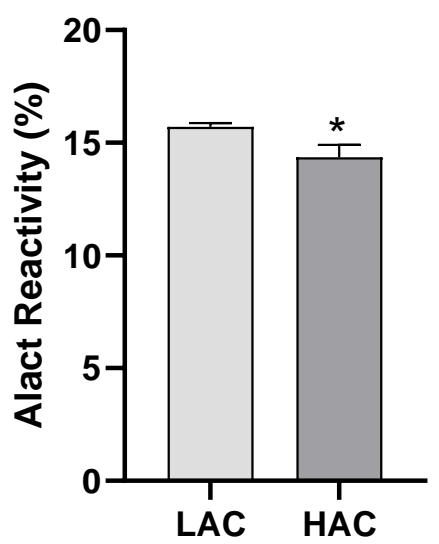

B)

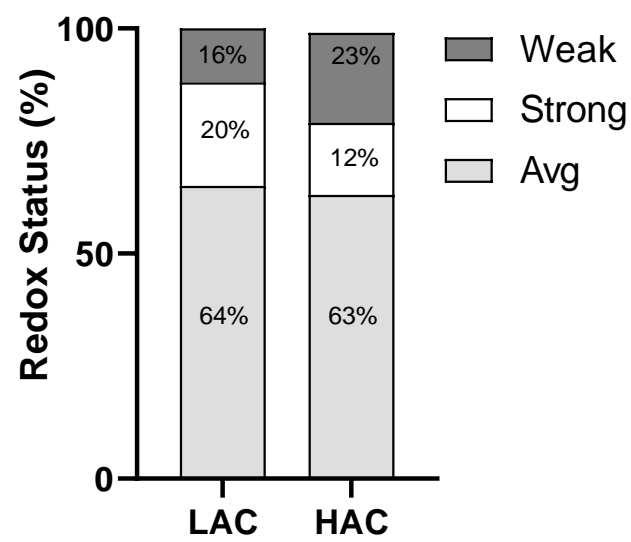

Figure 2: Effect of alcohol on Alcat reactivity and redox status. (A) Participants with a low alcohol consumption (LAC; $\mathrm{n}=813$ ) demonstrated an overall higher Alcat sensitivity, than the high alcohol consumption group (HAC, $\mathrm{n}=32$ ). Data are shown as the group average $\pm S E M$; asterisks indicate significance with significance being determined through an unpaired t-test $(\mathrm{p}=0.045)$. (B) The comparison of the percentage for Average (63\% vs 64\%), Strong (12\% vs $20 \%$ ), and weak (23\% vs 16\%) redox status of HAC participants against LAC participants. Dependence was examined through a chi-squared analysis $(\mathrm{p}=0.07)$.

\section{Effect of smoking on MND and Redox status distribution}

There was a larger number of identified MND in the smokers group, comparative to the nonsmokers, while AOD and Alcat reactivity did not differ between two groups. Unpaired t-test determined that the average number of MND was marginally higher $(p=0.09)$ (Fig. 3, A). Percentage of strong redox responses was higher (23\% vs $15 \%$ ) in the non-smokers group, while the percentage of the weak responses was higher in the smokers' group (21\% vs $11 \%)$. According to the chi-squared test, smoking had a marginally negative effect on immune redox function of the tested subjects $(\mathrm{p}=0.09)($ Fig 3, C).

A)

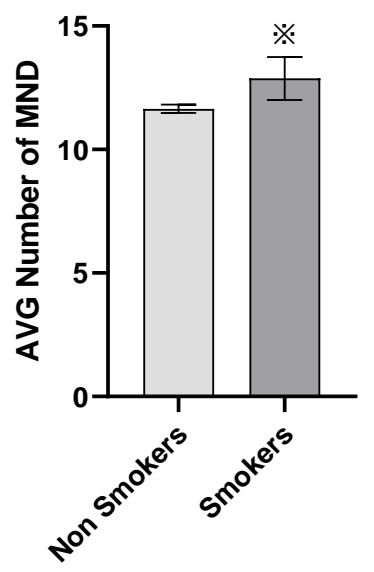

B)

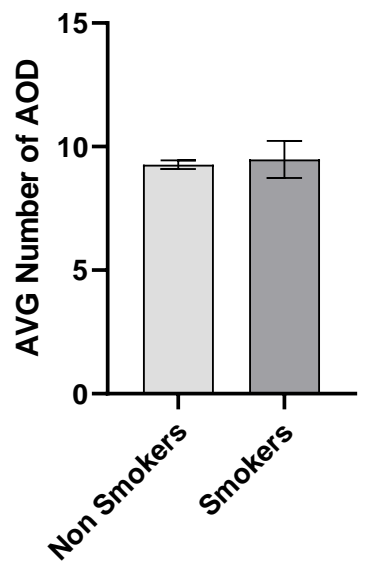

C)

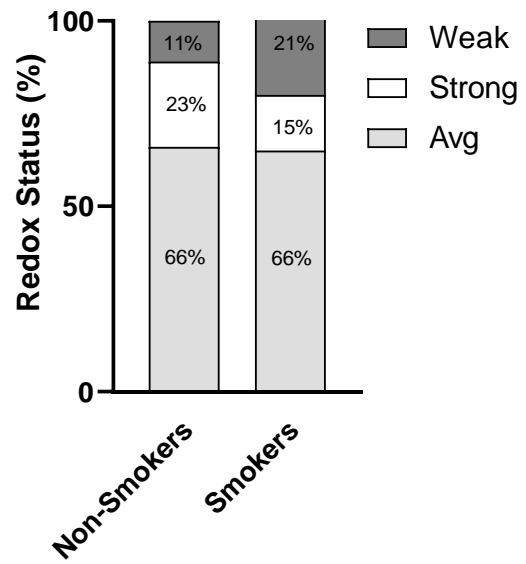

Figure 3. Effect of smoking on MND, AOD and food reactivity responses. Participants who were smokers $(n=814)$ demonstrated overall higher Alcat sensitivity, than Non-Smokers $(n=48)$. Unpaired t-tests were performed on (A) MND and (B) AOD, data are shown as the group average \pm SEM; reference marks $(※)$ indicate a marginal difference, with significance being determined through an unpaired $\mathrm{t}$-test $(\mathrm{p}$ $=0.09$ and 0.77 , respectively). (C) The comparison of the percentage for Average (66\% vs 66\%), Strong (15\% vs $23 \%$ ), and weak ( $21 \%$ vs $11 \%$ ) redox status of smokers compared to non-smokers. Dependence was examined through a chi-squared analysis $(\mathrm{p}=0.09)$. 
Effect of diet on redox status, micronutrient and antioxidant deficiencies, and food reactivity responses

According to the questionnaires' answers, all subjects were divided into 2 groups. The diet of the first group (healthy diet) consisted of primarily whole fresh foods including fruits, vegetables, nuts; while the (poor diet) group reported high consumption of sweets, fried, frozen and starchy foods. Mean values for micronutrient and antioxidant deficiency were significantly higher in the poor diet group as compared to the healthy diet group with p-values of 0.0017 and 0.0395 , respectively (Fig 4, A and B). The effect of the diet had no statistically significance on the food reactivity responses between the two groups. Assessment of the redox status in both groups showed higher percentage of the strong redox responses and showed lower percentage of the weak redox responses in the healthy diet group (Fig 4, C).

A)

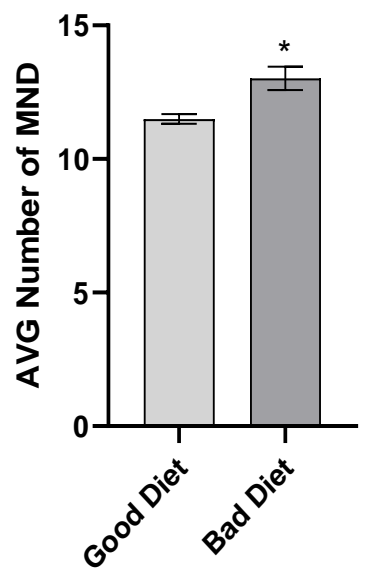

B)

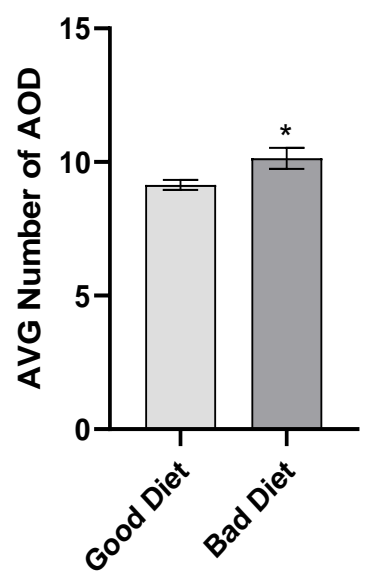

C)

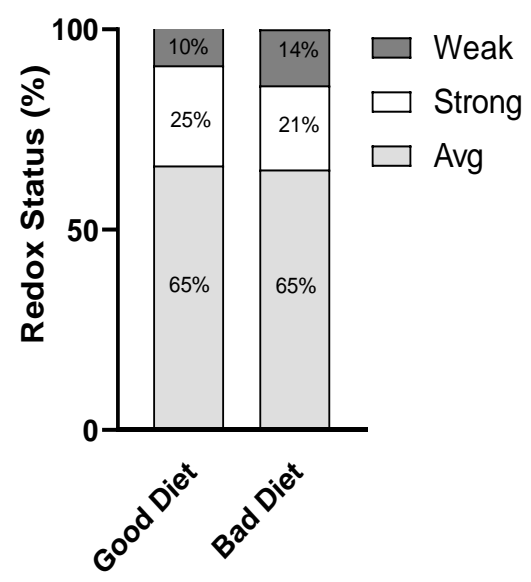

Figure 4. Effect of diet on MND, AOD and food reactivity responses. Participants who had a poor diet $(n=123)$ demonstrated overall higher Alcat sensitivity, than those who reported having a healthy diet ( $\mathrm{n}=739$ ). (A) MND and (B) AOD, data are shown as the group average \pm SEM; asterisks indicate significance determined through an unpaired t-test $(\mathrm{p}=0.0017$ and 0.0395 , respectively). (C) The comparison of the percentage for Average (65\% vs 65\%), Strong (25\% vs 21\%), and weak (10\% vs $14 \%$ ) redox status of participants with a healthy diet compared against those with a poor diet. Dependence was examined through a chi-squared analysis $(\mathrm{p}=0.6)$.

Effect of GI health on redox status, micronutrient and antioxidant deficiencies, and food reactivity responses

Group of patients with reported active GI issues demonstrated significantly higher percent of food reactivity than the group without GI issues $(\mathrm{p}=0.0048)$ as analyzed by unpaired t-test (Fig. 5, A). The mean value of micronutrient and antioxidant deficiencies was not significantly different between two groups. Percentages of strong and weak redox responses were $18 \%$ and $9 \%$, respectively in the healthy subjects, as compared to $14 \%$ and $15 \%$ in the group with GI issues (Fig $5, \mathrm{~B})$. 
A)

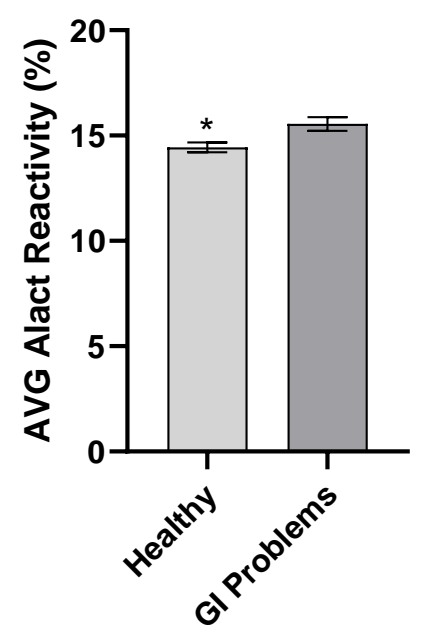

B)

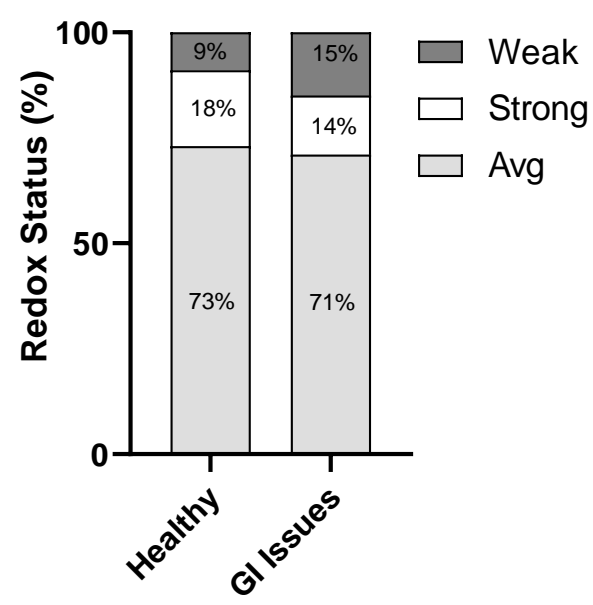

Figure 5. Effect of GI health on the number of micronutrient and antioxidant deficiencies. Participants who had GI issues $(n=215)$ were compared against participants who had a healthy GI system $(\mathrm{n}=232)$. (A) Alcat Reactivity data are shown as the group average \pm SEM; asterisks indicate significance, with significance being determined through an unpaired t-test $(\mathrm{p}=0.0048)$. (C) The comparison of the percentage for Average ( $73 \%$ vs $71 \%$ ), Strong (18\% vs $14 \%$ ), and Weak (9\% vs $15 \%$ ) redox status of participants with good gastrointestinal health compared against those with bad gastrointestinal health. Dependence was examined through a chi-squared analysis $(\mathrm{p}=0.36)$.

Age effect on redox status, micronutrient and antioxidant deficiencies, and food reactivity responses

According to the unpaired t-test,mean values of identified micronutrient deficiencies were statistically significantly higher $(p=0.0002)$ in the younger group (18 to 50 years old $)$ than in the age group over 50 years old (Fig. 6, A). The same trend was observed for antioxidant deficiencies; however, the difference was not statistically significant $(\mathrm{p}=0.0719)$ (Fig. 6, B). No significant differences were found in the redox status distribution between the two groups.

A)

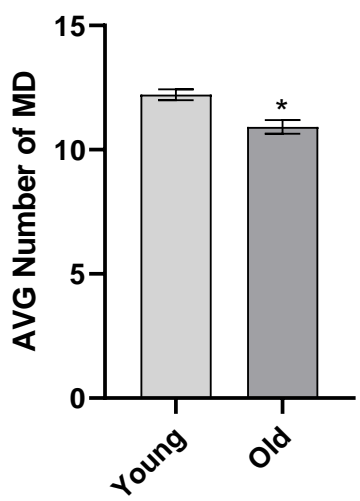

B)

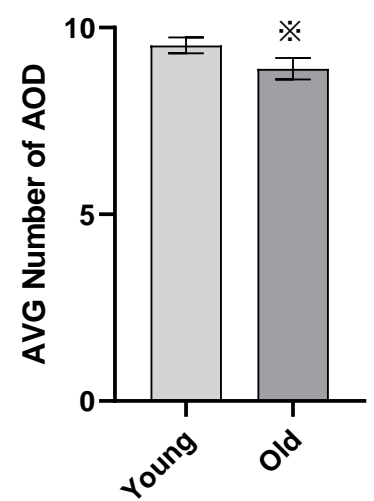

Figure 6. Effect of age on the number of micronutrient and antioxidant deficiencies. Alcat sensitivity of older participants $(n=332)$ was compared younger participants ( $n=531)$. (A) MND and (B) AOD data are shown as the group average \pm SEM; asterisks indicate statistical significance while a reference mark $(※)$ indicates marginal significance. Significance was determined through an unpaired ttest $(\mathrm{p}=0.0002$ and 0.0719 , respectively). 


\section{DISCUSSION}

Presently there is a great interest in investigating the role of nutrition in the prevention and possible cure of variety of medical symptoms and conditions. Laboratory serum tests are available for testing individual micronutrients and are generally used to confirm suspected micronutrient deficiencies. However, those tests do not reveal information about intracellular availability of the tested micronutrients. In addition, these tests are reporting results based on population averages, not on the functional cell responses of the tested individual. Intracellular nutrient status is probably more relevant than serum testing because it reflects more stable micronutrient levels over longer time periods than serum levels and reflects individual needs of the individual which may differ markedly from the general population for a number of reasons; such as, infection, pregnancy or other stressors, transport efficiency, metabolic function, etc. Thus, the balance of intra- and extracellular levels depends on a few factors, including the physiology of cellular transport mechanisms and the individual genetic background. Therefore, it is important to include the patient's serum depot into the functional assessment of micronutrient levels.

Lymphocyte metabolic activity is a normal and desirable response following mitogenic stimulation, therefore, provides a functional assessment that reflects the individual's requirements for optimal cellular functionality both of immune and other (non-immune) somatic cells. In the present study, we investigated the newly developed CSS cellular assays that use patient leucocytes suspended in the patient own serum to functionally assess micronutrient deficiencies in patients with a variety of biological backgrounds, medical history, and lifestyle. The present study, thus, investigated whether micronutrient deficiencies correlated with the changes of the immune redox status of patients.

Another important question explored in this study, is an effect of the biologic background and the lifestyle of patients on micronutrients and antioxidant deficiencies and overall immune response status. It was found that the higher number of micronutrient deficiencies correlates with the lower redox status of the tested patients. It was also found that lifestyle factors, like a poor diet, alcohol consumption, and tobacco smoking correlated with the increased micronutrient deficiencies and the ability of the immune system to resist oxidative stress. Also, biological factors such as age, influence micronutrient deficiencies and immune redox status of patients. Older subjects tend to have a higher incidence of infection and each episode lasts longer compared to younger individuals. For these reasons, several attempts have been made to study the immune system at various ages and to seek ways by which immunity can be enhanced by diet and nutritional supplements [13],[14]. The findings of this study were somewhat controversial to the existing belief that diseases and chronic conditions are an expected process of aging. There was no significant difference in the identified antioxidant deficiencies and Alcat reactivity between the young and older groups. Interestingly, the number of micronutrient deficiencies was lower in the older group. Since nutritional needs of each individual closely related to metabolic and physical activity, a condition of gastrointestinal permeability, taken medications and other patient-specific factors, it makes sense that an older population has generally lower metabolic activity rates and at the same time they are more conscious about micronutrient supplementation. These data are presenting the scientific rational for making a shift to more individualized approach that takes in consideration patients' age, activity status, physiological and genetic background, in selection of single or multiple micronutrients to reduce risk of chronic diseases and to improve overall health of patients. 
Oxidative stress occurs when the generation of ROS exceeds the ability of the body's antioxidant defense system to neutralize them. Increased oxidative stress and chronic inflammation are associated with the initiation and progression of most chronic human diseases such as cancer, heart disease, arthritis, diabetes, Alzheimer's disease, and Parkinson's disease. Therefore, attenuation of these two biological processes is of high importance. Several randomized, doubleblind, and placebo-controlled clinical studies of individual antioxidants in high-risk population resulted in controversial effects: from no effect, beneficial effects, to harmful effects [15], [16]. Close review of these studies raises fundamental questions whether these clinical trials took into consideration the status of oxidative stress and chronic inflammation that are elevated in high-risk populations. Therefore, an application of accurate methods for evaluation of patients' immune responses, as well as effects of single antioxidants on patients' immune status, are essential for the design of safe antioxidant therapies.

Alcoholism is one of the main medical conditions leading to antioxidant imbalance, as a result of an increased synthesis of reactive oxygen species, and an impaired antioxidant response triggered by ethanol metabolism. The ability of acute and chronic alcohol intake to increase ROS production, and potentiate cellular damage, has been demonstrated in a number of cells, systems, and species, including humans[17],[18]. Using our functional cellular assays, we have demonstrated a clear effect of the high alcohol consumption on the ability of patients' immune system to overcome oxidative stress. Also, we were able to identify and select the most efficient antioxidants for each tested individual. These data demonstrate suitability of our newly developed cellular assays for the accurate evaluation of the antioxidant efficacy of patients' immune system and for selection of the best therapeutic candidates.

Many gastrointestinal disorders with compromised microbiota in the gut may interfere with nutrient availability and impaired stimulation of gastrointestinal immune response. Under certain conditions, the immune system can produce toxic chemicals that play an important role in the initiation and progression of chronic diseases as well as cause autoimmune diseases. Gastrointestinal allergic disease includes a variety of adverse, food antigen-driven, immunemediated diseases [19]. Although, these diseases vary mechanistically; common elements include a breakdown of immunologic tolerance and an impaired mucosal barrier [20]. It was also recently reported that IFN- $\gamma$ produced by $\mathrm{T}$ cells directly induces intestinal stem cell death upon inflammation-induced intestinal injury [21]. These pathways are influenced by diverse factors such as diet, infections, exposure to antibiotics and chemicals, GI microbiome composition, and genetic and epigenetic elements. The difficulty of diagnosing food sensitivity which is not IgE mediated is well known. The Alcat test was developed to identify inflammation-triggering foods using WBC as a sensitive functional tool to identify those triggers that underlie such non- $\operatorname{IgE}$ mediated reactions to foods and chemicals. Using Alcat and CNA functional cellular assays we were able to identify nutritional deficiencies as well as overall immune status in patients with and without gastrointestinal issues. We also determined food sensitivity responses and compared them between two groups. We found significantly higher number of food reactivity responses in the group with gastrointestinal issues, than in patients with the healthy gut. Therefore, an accurate identification and elimination of inflammation-triggering foods can be a valuable tool in prevention and treatment of gastrointestinal disorders. 


\section{CONCLUSION}

These data clearly indicate direct correlation between patients' nutrient deficiencies and their immune status. Also, data suggests that high food sensitivity is associated with higher nutrient deficiency and lower immune redox status. Further studies can assess whether the effects of an addition of the identified deficient micronutrients into the patient diet and simultaneous restriction of the identified reactive foods may result in significant improvement of patients' redox status and their overall health condition. Preventive nutrition is a critical component not only of preventive medicine, but also of therapeutic medicine. Individualized nutrition is a very cost-effective way of decreasing mortality and improving overall individual functioning. Therefore, adequate and individualized nutritional intervention must be identified and implemented into the patients' regimen.

List of abbreviations: CSS, Cell Science Systems; CNA, cellular nutritional assays; SI, stimulation index; ROS, reactive oxygen species; DNA, deoxyribonucleic acid; HIPAA, health insurance portability and accountability act; GI, gastrointestinal; AOP, antioxidant protection assay, MN, micronutrient assay; AOD, antioxidant deficiencies; MND, micronutrient deficiencies; ANOVA, analysis of variance; RS, redox status; AvgAR, average percent of reactivity; WR, weak reaction; AR, average reaction; SR, strong reaction; SEM, standard error of the mean; HAC, high alcohol consumption; LAC, low alcohol consumption; ROS, reactive oxygen species; IFN- $\gamma$, interferon gamma; IgE, immunoglobulin E; WBC, white blood cell;

Competing interests: There are no conflicts of interests to declare.

Authors' contributions: R.D. and I.S. designed the research. R.D. supported the research. I.S. conducted the research, performed statistical analysis and wrote the manuscript. D.A. helped preform statistical analysis and format the manuscript. I.S. had primary responsibility for the final content. All authors read and approved the final version of the manuscript.

Acknowledgements and Funding: We are deeply grateful to Designs for Health, Inc. for providing micronutrients and antioxidants used in the research. Additionally, we thank the members of CSS laboratory for their technical assistance with the testing performance.

\section{REFERENCES}

1. Alpert PT: The Role of Vitamins and Minerals on the Immune System. Home Health Care Manag Pract. 2017, 29:199-202.

2. Lee GY, Han SN: The Role of Vitamin E in Immunity. Vol. 10, Nutrients. NLM (Medline); 2018, 10: 1614.

3. Carr AC, Maggini S: Vitamin C and immune function. Vol. 9, Nutrients. MDPI AG; 2017, 9: 1211.

4. Oh J, Choi R, Park HD, Lee H, Jeong BH, Park HY, et al.: Evaluation of vitamin status in patients with pulmonary tuberculosis. J Infect. 2017, 74:272-80.

5. Cunningham-Rundles S, McNeeley DF, Moon A: Mechanisms of nutrient modulation of the immune response. J Allergy Clin Immunol [Internet]. 2005 Jun 1 [cited 2019 Dec 19];115(6):1119-28. Available from: https://www.sciencedirect.com/science/article/abs/pii/S0091674905012741 
6. Victor VM, De la Fuente M: N-acetylcysteine improves in vitro the function of macrophages from mice with endotoxin-induced oxidative stress. Free Radic Res. 2002, 36:33-45.

7. Haase H, Rink L: The immune system and the impact of zinc during aging. Vol. 6, Immunity and Ageing. 2009. 6

8. De la Fuente M: Effects of antioxidants on immune system ageing. Eur J Clin Nutr. 2002, 56(Suppl 3): 5-8.

9. Hughes DA: Effects of dietary antioxidants on the immune function of middle-aged adults. Proc Nutr Soc. 1999, 58:79-84.

10. Maggini S, Pierre A, Calder PC: Immune function and micronutrient requirements change over the life course. Vol. 10, Nutrients. MDPI AG; 2018.

11. Marcos A, Nova E, Montero A: Changes in the immune system are conditioned by nutrition. Eur J Clin Nutr. 2003, 57(Suppl 1):66-9.

12. Mukaro VR, Costabile M, Murphy KJ, Hii CS, Howe PR, Ferrante A: Leukocyte numbers and function in subjects eating n-3 enriched foods: selective depression of natural killer cell levels. Arthritis Res Ther [Internet]. 2008 [cited 2019 Dec 19];10(3):R57. Available from: http://www.ncbi.nlm.nih.gov/pubmed/18477409

13. Chandra RK: Nutrition, immunity and infection: From basic knowledge of dietary manipulation of immune responses to practical application of ameliorating suffering and improving survival. Proc Natl Acad Sci U S A. 1996, 93:14304-7.

14. Wu D, Lewis ED, Pae M, Meydani SN: Nutritional modulation of immune function: Analysis of evidence, mechanisms, and clinical relevance. Vol. 10, Frontiers in Immunology. Frontiers Media S.A.; 2019.

15. Lonn E: Effects of long-term vitamin E supplementation on cardiovascular events and cancer: A randomized controlled trial. J Am Med Assoc. 2005, 293:1338-47.

16. Weinberg RB, VanderWerken BS, Anderson RA, Stegner JE, Thomas MJ: Pro-oxidant effect of vitamin $\mathrm{E}$ in cigarette smokers consuming a high polyunsaturated fat diet. Arterioscler Thromb Vasc Biol [Internet]. 2001 Jun [cited 2019 Dec 19];21(6):1029-33. Available from: http://www.ncbi.nlm.nih.gov/pubmed/11397715

17. Cederbaum AI, Lu Y, Wu D: Role of oxidative stress in alcohol-induced liver injury. Archives of Toxicology. 2009, 83:519-48.

18. Silva CS, Portari GV., Vannucchi H: Antioxidant Treatment and Alcoholism. In: Molecular Aspects of Alcohol and Nutrition: A Volume in the Molecular Nutrition Series. Elsevier Inc.; 2015, 119-31.

19. Saavedra-Delgado AM, Metcalfe DD: Interactions between food antigens and the immune system in the pathogenesis of gastrointestinal diseases. Ann Allergy [Internet]. $1985 \mathrm{Nov}$ [cited 2019 Nov 27];55(5):694-702. Available from: http://www.ncbi.nlm.nih.gov/pubmed/3904541

20. Azouz NP, Rothenberg ME: Mechanisms of gastrointestinal allergic disorders. Journal of Clinical Investigation. American Society for Clinical Investigation; 2019, 129:1419-30.

21. Kretzschmar K, Clevers H: IFN- $\gamma$ : The T cell's license to kill stem cells in the inflamed intestine. Sci Immunol [Internet]. 2019 Dec 6 [cited 2019 Dec 16];4(42):eaaz6821. Available from: http://immunology.sciencemag.org/lookup/doi/10.1126/sciimmunol.aaz6821 